December 1996, Final Version March 1997

Preprint TECHNION-PH-96-23

To be published in Physical Review D

MPI-PhT/96-126

\title{
Cherenkov radiation by massless neutrinos in a magnetic field
}

\author{
Ara N. Ioannisian \\ Yerevan Physics Institute, Alikhanyan Br. 2, Yerevan, 375036, Armenia \\ and Department of Physics, Technion-Israel Institute of Technology, Haifa 32000, Israel \\ Georg G. Raffelt \\ Max-Planck-Institut für Physik (Werner-Heisenberg-Institut), Föhringer Ring 6, 80805 München, Germany
}

(8 December 1996)

\begin{abstract}
We calculate the Cherenkov process $\nu \rightarrow \nu \gamma$ in the presence of a homogeneous magnetic field. The neutrinos are taken to be massless with only standard-model couplings. The magnetic field fulfills the dual purpose of inducing an effective neutrino-photon vertex and of modifying the photon dispersion relation such that the Cherenkov condition $\omega<|\mathbf{k}|$ is fulfilled. Our effect is closely related to photon splitting that occurs in magnetic fields and that may be astrophysically important in the strong magnetic fields of pulsars. It is also closely related to magnetic-field enhanced radiative decays $\nu \rightarrow \nu^{\prime} \gamma$ that have been extensively discussed in the recent literature. In the appropriate limits we agree with these results, but we disagree with earlier explicit calculations of the Cherenkov process. For a field strength $B_{\text {crit }}=m_{e}^{2} / e=4.41 \times 10^{13}$ Gauss and for $E=2 m_{e}$ the Cherenkov rate is about $6 \times 10^{-11} \mathrm{~s}^{-1}$ and thus too small to be of practical importance for pulsar physics.
\end{abstract}

PACS numbers: 13.15.+g, 14.60.Lm, 97.10.Ld, 97.60.Gb

\section{INTRODUCTION}

In many astrophysical environments the absorption, emission, or scattering of neutrinos occurs in dense media or in the presence of strong magnetic fields [1]. Of particular conceptual interest are those reactions which have no counterpart in vacuum, notably the plasmon decay $\gamma \rightarrow \bar{\nu} \nu$ and the Cherenkov process $\nu \rightarrow \nu \gamma$. These reactions do not occur in vacuum because they are kinematically forbidden and because neutrinos do not couple to photons. In the presence of a medium or $B$-field, neutrinos acquire an effective coupling to photons by virtue of intermediate charged particles. Also, neutrinos may have anomalous electromagnetic interactions, for example induced by a magnetic dipole moment. In addition, media or external fields modify the dispersion relations of all particles so that phase space is opened for neutrinophoton reactions of the type $1 \rightarrow 2+3$.

The plasma process $\gamma \rightarrow \bar{\nu} \nu$ was first studied by Adams, Ruderman, and Woo [2] and Zaidi [3] in order to calculate stellar energy losses into neutrinos. The $\nu-\gamma$ coupling is enabled by the presence of the electrons of the background medium, and the process is kinematically allowed because the photons acquire essentially an effective mass. The plasma process is the dominant source for neutrinos in many types of stars and thus is of great practical importance in astrophysics [1]. For that reason it also lends itself to deriving astrophysical limits on anomalous electromagnetic neutrino couplings which provide an additional contribution to the $\nu$ - $\gamma$-vertex and thus to the emission rate [1, 1, 5].
The presence of a magnetic field induces an effective $\nu$ - $\gamma$-coupling which contributes to the $\gamma \rightarrow \bar{\nu} \nu$ reaction. The resulting decay rate was calculated by Galtsov and Nikitina [6], Skobelev [7], and DeRaad, Milton, and Hari Dass [8], assuming that phase space is opened by a suitable medium- or field-induced modification of the photon refractive index.

If neutrinos are exactly massless as we will always assume, and if medium-induced modifications of their dispersion relation can be neglected, the photon decay $\gamma \rightarrow$ $\bar{\nu} \nu$ is kinematically possible whenever the photon four momentum $k=(\omega, \mathbf{k})$ is time-like, i.e. $k^{2}=\mathbf{k}^{2}-\omega^{2}<0$. . Often the dispersion relation is expressed by $|\mathbf{k}|=n \omega$ in terms of the refractive index $n$. In this language the photon decay is kinematically possible whenever $n<1$. In stellar plasmas this condition is usually satisfied, leading to the great practical importance of the plasma decay process for the physics of stars.

Even in a normal plasma there are electromagnetic excitations which fulfill the opposite condition $n>1$, namely the longitudinal plasmons or Langmuir waves $\gamma_{\mathrm{L}}$ which do not exist in vacuum. Their dispersion relation $\omega=f(\mathbf{k})$ "crosses the light cone" at a certain momentum $\mathbf{k}_{c}$ so that $\mathbf{k}^{2}-\omega^{2}<0$ for $|\mathbf{k}|<\left|\mathbf{k}_{c}\right|$ and $\mathbf{k}^{2}-\omega^{2}>0$ for $|\mathbf{k}|>\left|\mathbf{k}_{c}\right|$. Thus there is phase space for the Cherenkov

\footnotetext{
${ }^{*}$ We always use the metric $\operatorname{diag}(-+++)$ in accordance with much of the literature on magnetic-field effects on electromagnetic couplings.
} 
process $\nu \rightarrow \nu \gamma_{\mathrm{L}}$. Based on the standard-model interactions with the electrons of the medium, Tsytovich [9] was the first to calculate this sort of process. It was later rediscovered by Oraevsky, Semikoz, and Smorodinsky [10], Sawyer [11], D'Olivo, Nieves, and Pal [12], and Hardy and Melrose [13]. It was claimed that an intense neutrino beam could be particularly effective at emitting Langmuir waves by virtue of a nonlinear feed-back mechansim (Bingham et al. 14]), with important consequences for supernova physics. Unfortunately, this spectacular claim is erroneous because it was based on the assumption of a spurious phase-coherence of the neutrino states - see Hardy and Melrose [15].

Neutrinos may also couple to the electromagnetic field by virtue of an anomalous magnetic dipole moment, a hypothesis advanced a long time ago to solve the solar neutrino problem by magnetically induced spin oscillations. With this motivation in mind, Radomski [16] calculated the magnetic-moment Cherenkov process, but unsurprisingly found it too small to reduce the solar neutrino flux by any significant amount. Later this process was reconsidered by Grimus and Neufeld [17] and Mohanty and Samal [18]. The latter group considered the interior of a supernova core where even transverse plasmons (photons) appear to have a space-like dispersion relation because it is dominated by the magnetic moments of the nucleons rather than the charges of the electrons. In this environment the phase space for the Cherenkov process is large, while there is none for the photon decay.

Independently of the nature of the photon dispersion relation the process $\nu \rightarrow \nu \gamma$ occurs at the interface of two media with different refractive indices (transition radiation). With the assumption of a neutrino magnetic dipole moment this process was recently studied by Sakuda and Kurihara [19] and Grimus and Neufeld [20].

We presently extend previous studies of the Cherenkov process to neutrinos propagating in an external magnetic field. Around pulsars, for example, field strengths around the critical value $B_{\text {crit }}=m_{e}^{2} / e=4.41 \times 10^{13}$ Gauss and perhaps even larger are thought to occur. The electron density is probably so small that the photon dispersion relation is dominated by the magnetic field. The Cherenkov condition is then satisfied for significant ranges of photon frequencies. In addition, the magnetic field itself causes an effective $\nu$ - $\gamma$-vertex by standard-model neutrino couplings to virtual electrons and positrons. Therefore, we study the Cherenkov effect entirely within the particle-physics standard model.

A detailed literature search fi reveals that even this process has been calculated earlier by Galtsov and

\footnotetext{
${ }^{\dagger}$ The literature on neutrino Cherenkov radiation and related processes is extremely scattered. Many of the papers quoted here have never been referenced in the other papers on the same topic. Therefore, it is quite possible that we have overlooked other relevant works.
}

Nikitina [6] and Skobelev [7]. However, we do not agree with their results, which also sheds doubt on their treatment of the $\gamma \rightarrow \bar{\nu} \nu$ process.

Our work is closely related to a recent series of papers by Gvozdev, Mikheev, and Vasilevskaya [21] and to papers by Skobelev [22] and Kachelriess and Wunner [23] who studied the neutrino radiative decay $\nu \rightarrow \nu^{\prime} \gamma$ in the presence of magnetic fields where $\nu$ and $\nu^{\prime}$ are different neutrino flavors which are assumed to mix. This process would proceed even in the absence of external fields or media. In our case of massless unmixed neutrinos the initial and final state in $\nu \rightarrow \nu \gamma$ is the same flavor and the process does not take place in vacuum. The role of the external field at modifying the $\nu$ - $\gamma$-vertex in our study is however similar to Refs. 21 23. In addition, for us it is crucial that the magnetic field modifies the photon dispersion relation. In their case the process is kinematically allowed anyhow, and it depends on the neutrino mass difference if neglecting the exact photon dispersion relation is justified. In the appropriate limits we agree with the results of Refs. 21, 23.

Our work is also related to the process of photon splitting that may occur in magnetic fields as discussed, for example, in Refs. 24,25]. In photon splitting the magnetic field also plays the dual role of providing an effective three-photon vertex which does not exist in vacuum, and of modifying the dispersion relation of the differently polarized modes such that $\gamma \rightarrow \gamma \gamma$ becomes kinematically allowed for certain polarizations of the initial and final states. In fact, photon splitting could be called "Cherenkov radiation by photons in magnetic fields."

We proceed in Sec. II by deriving a general expression for the Cherenkov rate, assuming a general $\nu$ - $\gamma$-vertex. In Sec. III we derive the standard-model effective vertex in the presence of a homogeneous magnetic field. In Sec. IV we calculate the Cherenkov rate on the basis of the magnetic-field modified photon dispersion relation. In Sec. $\mathrm{V}$ we summarize our findings.

\section{CHERENKOV RADIATION}

Beginning with a general discussion of the Cherenkov process $\nu(p) \rightarrow \nu\left(p^{\prime}\right) \gamma(k)$ we note that in terms of the matrix element $\mathcal{M}$ the transition rate is

$$
\Gamma=\frac{1}{(2 \pi)^{2}} \frac{1}{2 E} \sum_{\text {pols. }} \int \frac{d^{3} \mathbf{k}}{2 \omega} \frac{d^{3} \mathbf{p}^{\prime}}{2 E^{\prime}} \delta^{4}\left(p-p^{\prime}-k\right)|\mathcal{M}|^{2} .
$$

Here, $p=(E, \mathbf{p}), p^{\prime}=\left(E^{\prime}, \mathbf{p}^{\prime}\right)$, and $k=(\omega, \mathbf{k})$ are the four momenta of the incoming neutrino, outgoing neutrino, and photon, respectively. The sum is over photon polarizations. It appears outside of the phase-space integrals because in general the photon refractive index depends on the photon polarization state.

With the identity $d^{3} \mathbf{p}^{\prime} / 2 E^{\prime}=\int d^{4} p^{\prime} \Theta\left(E^{\prime}\right) \delta\left(p^{2}\right)$ we may integrate over $\delta^{4}\left(p-p^{\prime}-k\right)$ and find 


$$
\begin{aligned}
\Gamma=\frac{1}{32 \pi^{2} E^{2}} & \sum_{\text {pols. }} \int \frac{|\mathbf{k}|}{\omega} d|\mathbf{k}| d \varphi d \cos \theta \\
& \times \delta\left(\frac{2 E \omega+\mathbf{k}^{2}-\omega^{2}}{2 E|\mathbf{k}|}-\cos \theta\right)|\mathcal{M}|^{2},
\end{aligned}
$$

where $\theta$ is the angle between the emitted photon and incoming neutrino. We have assumed that the neutrino dispersion relation is precisely light-like so that $p^{2}=0$ and $E=|\mathbf{p}|$. The integration over the azimuthal photon directions $\varphi$ is not yet carried out because the photon dispersion relation need not be isotropic.

The $\delta$-function constrains the photon emission angle to have the value

$$
\cos \theta=n^{-1}\left[1+\left(n^{2}-1\right) \frac{\omega}{2 E}\right],
$$

where we have used the photon refractive index $n=k / \omega$. Because it is not isotropic, this opening angle of the Cherenkov "cone" actually depends on the azimuthal direction $\varphi$.

In a magnetic field the photon refractive index is not isotropic, and it depends on the photon polarization. According to Adler's classic paper [24] there are two eigenmodes of photon propagation, one with the polarization vector parallel $(\|)$ and one perpendicular $(\perp)$ to the plane containing $\mathbf{k}$ and $\mathbf{B}$. f index in the form

$$
n_{\|, \perp}=1+\frac{\alpha}{4 \pi} \eta_{\|, \perp} \sin ^{2} \beta,
$$

where $\beta$ is the angle between $\mathbf{k}$ and $\mathbf{B}$. The numerical coefficients $\eta_{\|, \perp}$ depend on $B, \omega$, and $\beta$. For $B=\mathcal{O}\left(B_{\text {crit }}\right)$ they are of order unity. Therefore, for all situations of practical interest we have $\left|n_{\|, \perp}-1\right| \ll 1$. This allows us to expand Eq. (3) to lowest order in $\alpha$,

$$
\cos \theta=1-\frac{\alpha}{4 \pi} \eta_{\|, \perp}\left(1-\frac{\omega}{E}\right) \sin ^{2} \beta .
$$

This result reveals that to lowest order the outgoing photon propagates parallel to the original neutrino direction.

Therefore, to lowest order the azimuthal dependence of Eq. (2) drops out, allowing us to perform both angular integrations explicitly. Moreover, to this order we do not need to distinguish between $\omega$ and $|\mathbf{k}|=$ $n \omega=\omega[1+\mathcal{O}(\alpha)]$. Therefore, to lowest order in $\alpha$ the Cherenkov rate Eq. (2) is found to be

$$
\Gamma=\frac{1}{16 \pi E^{2}} \int_{0}^{\omega_{\max }} d \omega \sum_{\text {pols. }}|\mathcal{M}|^{2} .
$$

Energy conservation requires $\omega<E$ so that $\omega_{\max } \leq E$. The photon dispersion relation "crosses the light cone"

\footnotetext{
${ }^{\ddagger}$ Our definition of $\|$ and $\perp$ is opposite to Adler's 24 who used the photon's magnetic-field vector to define the polarization.
}

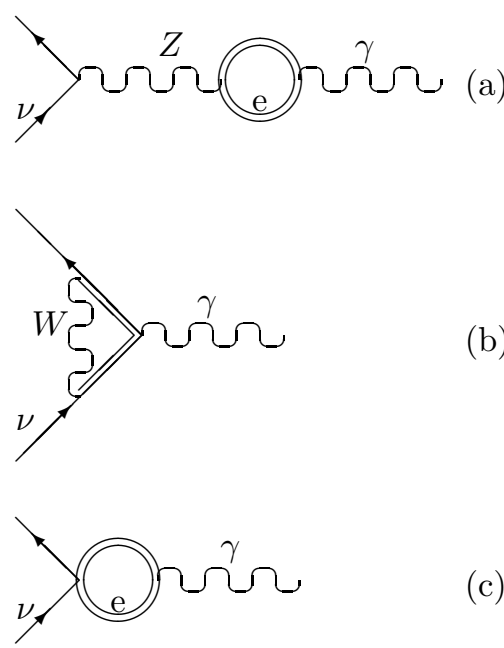

FIG. 1. Neutrino-photon coupling in an external magnetic field. The double line represents the electron propagator in the presence of a $B$-field. (a) $Z$ - $A$-mixing. (b) Penguin diagram (only for $\nu_{e}$ ). (c) Effective coupling in the limit of infinite gauge-boson masses.

at some frequency $\omega_{c}$ so that the Cherenkov condition is only satisfied for $0<\omega<\omega_{c}$. Therefore, $\omega_{\max }=$ $\min \left(E, \omega_{c}\right)$.

\section{THE NEUTRINO-PHOTON-VERTEX}

In a magnetic field, photons couple to neutrinos by the amplitudes shown in Figs. 1(a) and (b). The electron propagator, represented by a double line, is modified by the field to allow for a nonvanishing coupling. It has been speculated that superstrong magnetic fields may exist in the early universe, but we limit our discussion to field strengths not very much larger than $B_{\text {crit }}=m_{e}^{2} / e$ which is the range thought to occur in pulsars. Therefore, while in principle similar graphs exist for $\mu$ and $\tau$ leptons, we may neglect their contribution. For the same reason we may ignore field-induced modifications of the gauge-boson propagators. Moreover, we are interested in neutrino energies very much smaller than the $W$ - and $Z$-boson masses, allowing us to use the limit of infinitely heavy gauge bosons and thus an effective four-fermion interaction,

$$
\mathcal{L}_{\mathrm{eff}}=-\frac{G_{F}}{\sqrt{2}} \bar{\nu} \gamma_{\mu}\left(1-\gamma_{5}\right) \nu \bar{E} \gamma^{\mu}\left(g_{V}-g_{A} \gamma_{5}\right) E
$$

Here, $E$ stands for the electron field, $\gamma_{5}=i \gamma^{0} \gamma^{1} \gamma^{2} \gamma^{3}$, $g_{V}=2 \sin ^{2} \theta_{W}+\frac{1}{2}$ and $g_{A}=\frac{1}{2}$ for $\nu_{e}$, and $g_{V}=$ $2 \sin ^{2} \theta_{W}-\frac{1}{2}$ and $g_{A}=-\frac{1}{2}$ for $\nu_{\mu, \tau}$. In our subsequent calculations we will always use $\sin ^{2} \theta_{W}=\frac{1}{4}$ for the weak mixing angle so that the vector coupling will identically 
vanish for $\nu_{\mu}$ and $\nu_{\tau}$. Anyhow, we will find that the axial coupling is far more important.

The $\nu$ - $\gamma$-vertex is then given by the amplitude shown in Fig. 1(c) for which we find

$$
\begin{aligned}
\mathcal{M} & =i \frac{e G_{F}}{\sqrt{2}} Z \varepsilon_{\mu} \bar{\nu} \gamma_{\nu}\left(1-\gamma_{5}\right) \nu \\
& \times \int \frac{d^{4} p}{(2 \pi)^{4}} \operatorname{Tr}\left[\gamma^{\mu} G(p) \gamma^{\nu}\left(g_{V}-g_{A} \gamma_{5}\right) G(p-k)\right]
\end{aligned}
$$

Here, $G(p)$ denotes the electron propagator in a magnetic field, $p$ the four momentum of the electron in the loop, and $k$ the four momentum of the photon line. Further, $\varepsilon$ is the photon polarization vector and $Z$ its wave-function renormalization factor. For the physical circumstances of interest to us, the photon refractive index will be very close to unity so that we will be able to use the vacuum approximation $Z=1$.

The matrix element Eq. (8) can be written in the form

$$
\mathcal{M}=-\frac{G_{F}}{\sqrt{2} e} Z \varepsilon_{\mu} \bar{\nu} \gamma_{\nu}\left(1-\gamma_{5}\right) \nu\left(g_{V} \Pi^{\mu \nu}-g_{A} \Pi_{5}^{\mu \nu}\right)
$$

where

$$
\begin{aligned}
& \Pi^{\mu \nu}(k)=-i e^{2} \int \frac{d^{4} p}{(2 \pi)^{4}} \operatorname{Tr}\left[\gamma^{\mu} G(p) \gamma^{\nu} G(p-k)\right], \\
& \Pi_{5}^{\mu \nu}(k)=-i e^{2} \int \frac{d^{4} p}{(2 \pi)^{4}} \operatorname{Tr}\left[\gamma^{\mu} G(p) \gamma^{\nu} \gamma_{5} G(p-k)\right] .
\end{aligned}
$$

Here, $\Pi$ is the photon polarization tensor or vector-vector (VV) response function in the magnetic field, while $\Pi_{5}$ is the vector-axial vector (VA) response function.

In the presence of the external field the electron propagation function $G\left(x_{1}, x_{2}\right)$ satisfies the equation

$$
\left[m_{e}+\gamma\left(\frac{\partial}{i \partial x_{1}}-e A\left(x_{1}\right)\right)\right] G\left(x_{1}, x_{2}\right)=\delta\left(x_{1}-x_{2}\right)
$$

which can be solved exactly by Schwinger's proper-time method [26]. For the case of a purely homogeneous magnetic field in the 3 -direction $\left(F_{12}=-F_{21}=B_{3}=B\right)$ the result is 26, 27

$$
G\left(x_{1}, x_{2}\right)=\Phi\left(x_{1}, x_{2}\right) \int \frac{d^{4} p}{(2 \pi)^{4}} e^{i p\left(x_{1}-x_{2}\right)} G(p) .
$$

Here,

$$
\Phi\left(x_{1}, x_{2}\right)=\exp \left[i e \int_{x_{2}}^{x_{1}} d y A(y)\right]
$$

and

$$
\begin{aligned}
& G(p)=i \int_{0}^{\infty} d s \exp \left[-i s\left(m_{e}^{2}+p_{\|}^{2}+\frac{\tan z}{z} p_{\perp}^{2}\right)\right] \\
& \times \frac{1}{\cos z}\left[\left(m_{e}-\gamma p_{\|}\right) e^{i \sigma_{3} z}-\frac{\gamma p_{\perp}}{\cos z}\right]
\end{aligned}
$$

where $z=e B s$. Note that $\sigma_{3} z=F_{\mu \nu} \sigma^{\mu \nu} z / 2 B$ with $\sigma^{\mu \nu} \equiv \frac{i}{2}\left[\gamma^{\mu}, \gamma^{\nu}\right]$. The $\|$ and $\perp$ decomposition of a four vector $a$ is defined by $a_{\|}=\left(a_{0}, 0,0, a_{3}\right)$ with a spatial part parallel to the external $B$-field and $a_{\perp}=a-a_{\|}=$ $\left(0, a_{1}, a_{2}, 0\right)$. In the absence of a magnetic field $(B \rightarrow 0)$ obviously $G(p)=\left(\gamma p+m_{e}-i 0\right)^{-1}$.

The photon polarization tensor implied by this result has been calculated in Refs. 24,27,28. Following Ref. 27] it is

$$
\begin{aligned}
\Pi^{\mu \nu}(k)= & \frac{e^{3} B}{(4 \pi)^{2}} \int_{0}^{\infty} d s \int_{-1}^{+1} d v \\
\times & \left\{e ^ { - i s \phi _ { 0 } } \left[\left(g^{\mu \nu} k^{2}-k^{\mu} k^{\nu}\right) N_{0}\right.\right. \\
& \left.-\left(g_{\|}^{\mu \nu} k_{\|}^{2}-k_{\|}^{\mu} k_{\|}^{\nu}\right) N_{\|}+\left(g_{\perp}^{\mu \nu} k_{\perp}^{2}-k_{\perp}^{\mu} k_{\perp}^{\nu}\right) N_{\perp}\right] \\
& \left.-e^{-i s m_{e}^{2}}\left(1-v^{2}\right)\left(g^{\mu \nu} k^{2}-k^{\mu} k^{\nu}\right)\right\}
\end{aligned}
$$

where

$$
\phi_{0}=m_{e}^{2}+\frac{1-v^{2}}{4} k_{\|}^{2}+\frac{\cos z v-\cos z}{2 z \sin z} k_{\perp}^{2} .
$$

Further,

$$
\begin{aligned}
& N_{0}=\frac{\cos z v-v \cot z \sin z v}{\sin z}, \\
& N_{\|}=-\cot z\left(1-v^{2}+\frac{v \sin z v}{\sin z}\right)+\frac{\cos z v}{\sin z}, \\
& N_{\perp}=-\frac{\cos z v}{\sin z}+\frac{v \cot z \sin z v}{\sin z}+2 \frac{\cos z v-\cos z}{\sin ^{3} z} .
\end{aligned}
$$

The $\|$ and $\perp$ decomposition of the metric is $g_{\|}=$ $\operatorname{diag}(-, 0,0,+)$ and $g_{\perp}=g-g_{\|}=\operatorname{diag}(0,+,+, 0)$.

The VA response function $\Pi_{5}$ has been calculated in Ref. [8]. However, their calculation contains several errors which require a reconsideration of $\Pi_{5}$. It is [8]

$$
\begin{aligned}
\Pi_{5}^{\mu \nu}(k)= & i \frac{e^{3}}{(4 \pi)^{2}} \int_{0}^{\infty} d s \int_{-1}^{+1} d v e^{-i s \phi_{0}} \\
\times & \left(\left(2 m_{e}^{2}+\frac{1-v^{2}}{2} k_{\|}^{2}\right) \widetilde{F}^{\mu \nu}-\left(1-v^{2}\right) k_{\|}^{\nu}(\widetilde{F} k)^{\mu}\right. \\
& \left.+R\left[k_{\perp}^{\mu}(k \tilde{F})^{\nu}-k_{\perp}^{2} \widetilde{F}^{\mu \nu}\right]\right\}
\end{aligned}
$$

where

$$
R=\frac{1-v \sin z v \sin z-\cos z \cos z v}{\sin ^{2} z}
$$

and $\widetilde{F}^{\mu \nu}=\frac{1}{2} \epsilon^{\mu \nu \rho \sigma} F_{\rho \sigma}$ with $\epsilon^{0123}=1$ is the dual of the field-strength tensor.

This result is not gauge invariant. However, one may integrate the first term under the integral by parts \& 8

$$
\begin{aligned}
\int_{0}^{\infty} d s\left(2 m_{e}^{2}\right. & \left.+\frac{1-v^{2}}{2} k_{\|}^{2}\right) e^{-i s \phi_{0}} \\
& =-2 i-\int_{0}^{\infty} d s k_{\perp}^{2} e^{-i s \phi_{0}} R .
\end{aligned}
$$


The first term on the r.h.s. does not depend on the mass of the particle in the loop. For the $Z$ - $A$-mixing amplitude (Fig. 1a) it cancels when we take into account all fermions from each generation according to the cancellation of the Adler anomaly in the Standard Model [29]. For the penguin diagram this term disappears when we take into account the exact $W$ propagator [30].

With these results we find for the VA response function

$$
\begin{aligned}
\Pi_{5}^{\mu \nu}(k) & =\frac{e^{3}}{(4 \pi)^{2} m_{e}^{2}}\left\{-C_{\|} k_{\|}^{\nu}(\widetilde{F} k)^{\mu}\right. \\
& \left.+C_{\perp}\left[k_{\perp}^{\nu}(k \widetilde{F})^{\mu}+k_{\perp}^{\mu}(k \widetilde{F})^{\nu}-k_{\perp}^{2} \widetilde{F}^{\mu \nu}\right]\right\}
\end{aligned}
$$

where

$$
\begin{aligned}
& C_{\|}=i m_{e}^{2} \int_{0}^{\infty} d s \int_{-1}^{+1} d v e^{-i s \phi_{0}}\left(1-v^{2}\right) \\
& C_{\perp}=i m_{e}^{2} \int_{0}^{\infty} d s \int_{-1}^{+1} d v e^{-i s \phi_{0}} R
\end{aligned}
$$

are dimensionless coefficients which are real for $\omega<2 m_{e}$, i.e. below the pair-production threshold.

\section{CHERENKOV RATE}

Armed with these results we may now turn to an evalulation of the rate for $\nu \rightarrow \nu \gamma$. It is easy to see that for both photon eigenmodes the parity-conserving part of the effective vertex $\left(\Pi^{\mu \nu}\right)$ is proportional to the small parameter $\left(n_{\|, \perp}-1\right)^{2} \approx(\alpha / 2 \pi) \eta_{\|, \perp} \sin ^{2} \beta$. It is important to note that the parity-violating part $\left(\Pi_{5}^{\mu \nu}\right)$ is not proportional to this small parameter for the $\|$ photon mode, while it is proportional to it for the $\perp$ mode.

It is interesting to compare this finding with the standard plasma decay process $\gamma \rightarrow \bar{\nu} \nu$ which is dominated by the VV vertex function. Therefore, in the approximation $\sin ^{2} \theta_{W}=\frac{1}{4}$ only the electron flavor contributes to plasmon decay. Here, we are in the opposite situation where the axial coupling to the electrons is the dominating one so that the Cherenkov rate is equal for (anti)neutrinos of all flavors.

For neutrinos which propagate perpendicular to the magnetic field, Eqs. (6), (9), and (22) lead to a Cherenkov emission rate of $\|$ photons of

$$
\Gamma=\frac{2 \alpha G_{F}^{2}}{(4 \pi)^{4}}\left(\frac{B}{B_{\text {crit }}}\right)^{2} \int_{0}^{\omega_{\max }} d \omega \omega^{4}\left(1-\frac{\omega}{E}\right)\left(\frac{C_{\|}}{2}-C_{\perp}\right)^{2} .
$$

We consider at first neutrino energies below the pairproduction threshold $E<2 m_{e}$. For $\omega<2 m_{e}$ the photon refractive index [24,31] always obeys the Cherenkov condition $n>1$ so that $\omega_{\max }=E$. Further, it turns out that in the range $0<\omega<2 m_{e}$ the expression $C_{\|} / 2-C_{\perp}$ depends only weakly on $\omega$ so that it is well approximated by its value at $\omega=0$. Therefore, Eq. (24) can be written in the form

$$
\begin{aligned}
\Gamma & \approx \frac{4 \alpha G_{F}^{2} E^{5}}{135(4 \pi)^{4}}\left(\frac{B}{B_{\text {crit }}}\right)^{2} h(B) \\
& =2.0 \times 10^{-9} \mathrm{~s}^{-1}\left(\frac{E}{2 m_{e}}\right)^{5}\left(\frac{B}{B_{\text {crit }}}\right)^{2} h(B),
\end{aligned}
$$

where

$$
h(B) \equiv \frac{9}{16}\left(C_{\|}-2 C_{\perp}\right)_{\omega=0}^{2} .
$$

Explicitly, this is found to be

$$
h(B)= \begin{cases}(4 / 25)\left(B / B_{\text {crit }}\right)^{4} & \text { for } B \ll B_{\text {crit }} \\ 1 & \text { for } B \gg B_{\text {crit }} .\end{cases}
$$

Evidently, even if the field strength is around the critical value, the Cherenkov rate is rather small.

Turning next to the case $E>2 m_{e}$ we note that in the presence of a magnetic field the electron and positron wavefunctions are Landau states so that the process $\nu \rightarrow \nu e^{+} e^{-}$becomes kinematically allowed. Therefore, neutrinos with such large energies will lose energy primarily by pair production rather than by Cherenkov radiation-for recent calculations see Refs. 32].

The Cherenkov effect $\nu \rightarrow \nu \gamma$ has been previously calculated in Refs. [6.77. However, they have not taken the neutral-current part into account so that their result applies only to $\nu_{e}$ for which the effective axial coupling $g_{A}=1$ was used instead of our $g_{A}=1-\frac{1}{2}=\frac{1}{2}$ which is a sum of the charged- and neutral-current contributions. Further, their final result is larger by a factor $2^{4} \pi$ relative to our Eq. (25).

We may also compare our $\nu \rightarrow \nu \gamma$ calculation with previous $\nu \rightarrow \nu^{\prime} \gamma$ ones [21 23]. To this end we note that in $\nu \rightarrow \nu^{\prime} \gamma$ the photon energy obeys $0<\omega<E$ if $\nu$ is ultrarelativistic, if $m_{\nu^{\prime}}=0$, and if one uses the vacuum photon dispersion relation. As this is the same range allowed in our case, there is no phase-space complication and we may compare our Eq. (25) with Eq. (15) of Ref. [23]. However, we must identify $\nu^{\prime}$ with $\nu$ which implies that we must drop their mixing-angle factors. Further, we must substitute $g_{A}=\frac{1}{2}$ for their value 1 to account for the neutral-current contribution. After these modifications our result is still a factor of 2 larger. This is explained, we believe, by their use of an unpolarized initial state of massive Dirac neutrinos while our neutrinos are always left-handed.

If $\nu^{\prime}$ is not taken to be massless, the $\nu \rightarrow \nu^{\prime} \gamma$ decay rate is reduced and vanishes for $m_{\nu^{\prime}}=m_{\nu}$. This, of course, is just our case of identical initial and final states where the Cherenkov rate by no means vanishes. This discrepancy is due to the use of the vacuum photon dispersion relation in Refs. 21 23. Using a consistent dispersion relation prevents this suppression effect because for ultrarelativistic initial neutrinos, the allowed range of photon energies will always be $0<\omega<E$. 


\section{SUMMARY AND CONCLUSIONS}

We have calculated the neutrino Cherenkov process $\nu \rightarrow \nu \gamma$ in a homogeneous magnetic field. The magnetic field provides an effective $\nu$ - $\gamma$-vertex, and it modifies the photon dispersion relation such that the Cherenkov condition is met for photon energies $\omega<2 m_{e}$. The neutrino emits primarily photons with a polarization vector parallel to the transverse component of the magnetic field (the I| propagation eigenmode), and the coupling is primarily due to the VA (pseudotensor) electromagnetic vertex function. We have corrected some errors of a previous calculation of this dominant term which had been studied in the context of the $\gamma \rightarrow \bar{\nu} \nu$ process in magnetic fields. We have also corrected errors in previous calculations of the Cherenkov process.

For neutrinos propagating transverse to the magnetic field, the Cherenkov rate is numerically given in Eq. (25). The strongest magnetic fields known in nature are near pulsars. However, they have a spatial extent of only tens of kilometers. Therefore, even if the field strength is as large as the critical one, most neutrinos escaping from the pulsar or passing through its magnetosphere will not emit Cherenkov photons. Thus, the magnetosphere of a pulsar is quite transparent to neutrinos as one might have expected.

\section{ACKNOWLEDGMENTS}

We are grateful to Gagik Grigorian, Jose Valle, and Daniel Wyler for useful discussions. We thank Alexander Kuznetsov and Nicolay Mikheev for pointing out a missing factor of 2 in Eq. (24) of our original manuscript. A.I. acknowledges the hospitality of the Max-Planck-Institut für Physik during a visit when this work was begun. This research was supported, in part, by the Deutsche Forschungsgemeinschaft grant SFB 375 (G.R.) and by the Lady Davis Trust (A.I.).

[1] G. G. Raffelt, Stars as Laboratories for Fundamental Physics (University of Chicago Press, Chicago, 1996).

[2] J. B. Adams, M. A. Ruderman, and C.-H. Woo, Phys. Rev. 129, 1383 (1963).

[3] M. H. Zaidi, Nuovo Cim. 40, 502 (1965).

[4] J. Bernstein, M. A. Ruderman, and G. Feinberg, Phys. Rev. 132, 1227 (1963).

[5] G. G. Raffelt, Astrophys. J. 365, 559 (1990). G. G. Raffelt and A. Weiss, Astron. Astrophys. 264, 536 (1992).

[6] D. V. Galtsov and N. S. Nikitina, Zh. Eksp. Theor. Fiz. 62, 2008 (1972) [Sov. Phys. JETP 35, 1047 (1972)].

[7] V. V. Skobelev, Zh. Eksp. Teor. Fiz. 71, 1263 (1976) [Sov. Phys. JETP 44, 660 (1976)].
[8] L. L. DeRaad Jr., K. A. Milton, and N. D. Hari Dass, Phys. Rev. D 14, 3326 (1976).

[9] V. N. Tsytovich, J. Exptl. Theoret. Phys. (U.S.S.R.) 45, 1183 (1963) [Sov. Phys. JETP 18, 816 (1964)].

[10] V. N. Oraevsky, V. B. Semikoz and Ya. A. Smorodinsky, Pis'ma Zh. Eksp. Theor. Fiz. 43, 549 (1986) [JETP Lett. 43, 709 (1986).

[11] R. F. Sawyer, Phys. Rev. D 46, 1180 (1992).

[12] J. D'Olivo, J. Nieves and P. Pal, Phys. Lett. B 365, 178 (1996).

[13] S. J. Hardy and D. B. Melrose, Publ. Astron. Soc. Aus. 13, 144 (1996).

[14] R. Bingham, J. M. Dawson, J. J. Su, and H. A. Bethe, Phys. Lett. A 193, 279 (1994).

[15] S. J. Hardy and D. B. Melrose, Langmuir Wave Generation Through a Neutrino Beam Instability, Astrophysical Journal, in press (1997). Preprint available at http://www.physics.usyd.edu.au/ stephen/work.htm

[16] M. Radomski, Phys. Rev. D 12, 2208 (1975).

[17] W. Grimus and H. Neufeld, Phys. Lett. B 315, 129, (1993).

[18] S. Mohanty and M. K. Samal, Phys. Rev. Lett. 77, 806 (1996).

[19] M. Sakuda, Phys. Rev. Lett. 72, 804 (1994). M. Sakuda and Y. Kurihara, Phys. Rev. Lett. 74, 1284 (1995).

[20] W. Grimus and H. Neufeld, Phys. Lett. B 344, 252 (1995).

[21] A. A. Gvozdev, N. V. Mikheev, and L. A. Vasilevskaya, Phys. Lett. B 289, 103 (1992); 292, 176 (1992); 313, 161 (1993); 321, 108 (1994); 323, 179 (1994); Phys. Rev. D 54, 5674 (1996). L. A. Vasilevskaya, A. A. Gvozdev, and N. V. Mikheev, Yad. Fiz. 55, 1029 (1992) [Sov. J. Nucl. Phys. 55, 579 (1992)]; Yad. Fiz. 57, 124 (1994) [Phys. At. Nucl. 57, 117 (1994)]; Yad. Fiz. 58, 712 (1995) [Phys. At. Nucl. 58, 654 (1995)].

[22] V. V. Skobelev, Zh. Eksp. Teor. Fiz. 108, 3 (1995) [JETP 81, 1 (1995)].

[23] M. Kachelriess and G. Wunner, Phys. Lett. B 390, 263 (1997).

[24] S. L. Adler, Ann. Phys. (N.Y.) 67, 599 (1971).

[25] S. L. Adler, E-print astro-ph/9601156. S. L. Adler and C. Schubert, Phys. Rev. Lett. 77, 1695 (1996). M. Mentzel, D. Berg, and G. Wunner, Phys. Rev. D 50, 1125 (1994). G. Wunner, R. Sang, and D. Berg, Astrophys. J. 455, L51 (1995). V. N. Baier, A. I. Milstein, and R. Zh. Shaisultanov, Phys. Rev. Lett. 77, 1691 (1996).

[26] J. Schwinger, Phys. Rev. 82, 664 (1951).

[27] W.-Y. Tsai, Phys. Rev. D 10, 2699 (1974).

[28] A. Minguzzi, Nuovo Cim. 7, 501 (1957).

[29] S. L. Adler, Phys. Rev. 177, 2426 (1969).

[30] D. Wyler and H. Simma, Nucl. Phys. B 344, 283 (1990).

[31] W.-Y. Tsai and T. Erber, Phys. Rev. D 10, 492 (1974); 12, 1132 (1975); Act. Phys. Austr. 45, 245 (1976).

[32] A. V. Borisov, A. I. Ternov, and V. Ch. Zhukovsky, Phys. Lett. B 318, 489 (1993). A. V. Kuznetsov and N. V. Mikheev, Phys. Lett. B 394, 123 (1997). 